\title{
PREVALENCE OF POSTNATAL DEPRESSION AND ASSOCIATED RISK FACTORS AMONG SOUTH ASIAN MOTHERS LIVING IN A NEWLY DEVELOPING COUNTRY
}

\author{
HASHIM AL SAYED MOHAMED ${ }^{1}$, MARYAM MOHAMED AL JABER ${ }^{2}$, ZAIDFAHIM AL-HAMADANI ${ }^{1}$, \\ HAMZEH YOUSEF KHMOUR ${ }^{1}$, BADRYIA ABDULLA AL LENJAWI ${ }^{3}$, JOSIA MARKUS SCHLOGL ${ }^{4}$
}

\begin{abstract}
${ }^{1}$ Department of Family Medicine, Weill Cornell Medicine-Qatar, Doha, Qatar. ${ }^{2}$ Department of Family Medicine, Primary Health Care Corporation, Doha, Qatar. ${ }^{3}$ Department of Nursing, Hamad Medical Corporation, Doha, Qatar. ${ }^{4}$ Department of Family Medicine, Weill Cornell Medicine-Qatar Doha, Qatar. Email: fmcc2000@gmail.com
\end{abstract}

Received: 17 May 2016, Revised and Accepted: 24 May 2016

\section{ABSTRACT}

Objective: Postnatal depression (PND) is a major health problem affecting mother, her child and family. Its prevalence and associated risk factors among South Asian mothers (SAM) living in Qatar remain unknown. This study is conducted in order to estimate the prevalence of PND among SAM in Qatar and to correlate risk factors contributing to the development of PND.

Methods: A total of (285) (SAM) females who were 6 months post-partum were interviewed as part of a prospective study conducted in primary health care centers in Qatar. PND symptoms were defined as present when subjects had an Edinburgh postnatal depression scale score of 10 or higher. Descriptive statistics were used for summarizing the study and outcome variables. The $\chi 2$ test and odds ratios with $95 \%$ confidence intervals were used for observation and quantifying the association between different variables. Multivariate binary logistic regression was used to identify the independent associated factors of PPD. $p \leq 0.05$ was considered significant. Variables included were age, occupation, education level, previous psychiatric history, comorbidities, history of depression during current pregnancy, history of anxiety during current pregnancy, number of previous pregnancies, strong social support, husband support, marital problem before pregnancy and ongoing marital problems during current pregnancy.

Results: The prevalence of PND among 285 respondents was 33.2\%. Several psychosocial risk factors were significantly associated with PND after multiple regression analysis including a history of depressive illness, anxiety, marital problems before delivery, a history of diabetes and asthma, history of congenital malformations, and lack of mother support. Other variables, including age, parity, education, occupation, and delivery type, were not significantly correlated $(\mathrm{p}=0.15-0.95)$, but marginally indicative of the risk of depressive symptoms.

Conclusion: This study showed a high rate of depressive symptoms among (SAM) 6 months post-partum. Future screening protocols must be employed at primary care level and hospital based clinics in Qatar to detect and treat post natal depression.

Keywords: Postnatal depression, South Asians, Primary health care, Doha, Edinburgh postnatal depression scale.

(C) 2016 The Authors. Published by Innovare Academic Sciences Pvt Ltd. This is an open access article under the CC BY license (http://creativecommons. org/licenses/by/4. 0/) DOI: http://dx.doi.org/10.22159/ajpcr.2016.v9i6.12900

\section{INTRODUCTION}

Qatar, one of the smallest and wealthiest countries in the world, is a newly developing country which relies mainly on foreign workforce for its development and prosperity. In general, foreign workers make up $80 \%$ of the population and the biggest portion of the population in Qatar are from South Asian countries, mainly India, Bangladesh, and Pakistan which comprises estimated at $45 \%$ of the population, whereas Qatari nationals represent only $20 \%$ of the population a minority [1]

Postnatal depression (PND) may range from mild self-limiting depression named postpartum blues to postnatal major depression and psychosis. Most women are affected by mood symptoms in postpartum periods (4-6 weeks after the child birth). PND is characterized by mood disturbance, mood instability, feeling of unhappiness, dysphoria, mental confusion, and weeping [2]. At the other end of the spectrum is postpartum psychosis, which is characterized by severely depressed mood, disorganized thinking, hallucination, and psychotic thoughts. PND is a public health problem leading to decreased quality of life and family tensions and may lead to abuse or neglect of the newborn. PND affects $10-15 \%$ of women [3] and is indeed under diagnosed and undertreated, especially in this part of the world where depression may be considered a normal part of the human cycle. The effects of PND go beyond the mother to affect partner and the child, as a result, it can be deemed a public health problem. In addition, severe PND may lead to maternal death, often by suicide as well as infanticide. Although the literature demonstrates that low- to middle-income countries face the greatest burden. However, any population may be faced with the challenge of PND including those going through rapid socioeconomic transitions where day to day pressures can lead to absenteeism of the partner for long hours or days during a critical time where the mother is at most vulnerable. The majority of expectant mothers in Qatar are routinely cared for in primary care setting and only complicated cases get referred to secondary care including those with gestational diabetes, pre-eclampsia, and previous history of abortions. During their followup, pregnant female get screened for anemia, gestational diabetes, and pre-eclampsia. However, during their 2 months and 6-month follow-up appointment, postnatal female does not get screened for PND whether at the primary care or secondary care. The prevalence of PND among South Asian mothers (SAM) living in Qatar is unknown, and screening for PND and its risk factors currently does not take place during the postnatal visit.

\section{METHODS}

This prospective study was carried out in Doha, Qatar, between March and December 2013. The goal of the study is to assess the prevalence of PND and to identify the correlated risk factors. Ethical approval of the study was guaranteed from the Medical Research Committee (\#11005) at Hamad Medical Corporation, Doha, Qatar. Informed consent was obtained from study participants. Data were coded to protect the confidentiality of participants, and information was extracted by the main researcher. Female participants were invited to participate from the 16 health centers covering the state of Qatar to ensure a random 
sample. The study participants were (SAM) females who signed an informed consent letter, delivered normal healthy child, and scheduled for routine well baby clinic appointment of the primary health care at 6 months postdelivery. All postnatal mothers were literate and did not require any assistance with the questionnaire. Postnatal mothers who gave birth to a baby with congenital diseases and malformations were not excluded from the study. The research questionnaire contains 22 closed-ended questions in the English language. The questionnaire is divided into three parts: The first part deals with sociodemographic variables, which include age, education level, and occupation; the second part contains obstetric history variables and past psychiatric history of the participant, comorbidities, satisfaction with child feeding pattern, history of depression during current pregnancy, history of anxiety during current pregnancy, number of previous pregnancies, strength of social support, husband support, marital problem before pregnancy and ongoing marital problems during current pregnancy. Edinburgh postnatal depression scale (EPDS) is the most widely used and culturally validated instrument [4]. It is a self-administered questionnaire consisting of 10 questions assessing the mother's feelings over the last 7 days. EPDS scores of more than 12 were the optimum cutoff scores for probable depression as validated in various ethnic minorities $[5,6]$. To fully describe the studied sample, scores of 0-9 indicates no risk of experiencing symptoms of PND, scores of 10-12 indicates minor/major risk of experiencing symptoms of PND (possible depression), and scores of 13 or above indicates a major risk of experiencing symptoms of PND (probable depression). A separate questionnaire was also added to identify the possible risk factors of PND. All data analysis was conducted using SPSS (20.0). Multiple imputations were used to treat the missing data. A negative binomial regression model was used to identify the association of the outcome (EPDS scores) at 2 weeks and 8 weeks, as well as each variable. The adjusted odds ratio (OR) and the 95\% confidence interval (CI) (95\% $\mathrm{CI}$ ) were reported, and a $\mathrm{p}=0.05$ was considered statistically significant.

\section{Sample size}

Using a CI of $95 \%(5 \% \mathrm{CI})$ and an estimated prevalence of $20 \%$ similar to studies in the region $[7,8]$, the sample size was calculated to be 285 . Study subjects were selected via random sampling from all 16 health centers covering Qatar. A proportional sample was calculated for each region and health center using the list of birth notifications relevant to each selected health center that was collected during the study period.

\section{Data analysis}

Data were coded, entered, and analyzed using SPSS, version 16.0. Continuous variables were described using descriptive statistics including mean and standard deviation (SD), and frequencies were used to describe categorical variables. Chi-squared test of independence was used to test the association between the dependent variable PND symptoms and each of the sociodemographic, social support, infant, maternal, and psychiatric health conditions. Fisher exact test was used for counts $<5$. OR was calculated with the $95 \%$ CIs to measure the risk of PND symptoms in relation to the studied risk factors. The model was estimated using the backward conditional logistic regression.

Adjusted OR was calculated for the significant predictors. $\mathrm{p}<0.05$ was considered statistically significant. Most important risk factors were identified using multiple logistic regression. Data were extracted for putative risk factors from participants medical charts along with previously validated questionnaire assessing social, financial support, and marital problems.

\section{RESULTS}

A total of 285 (SAM) mothers took part in the study, representing a response rate of $94.8 \%$. Around one-third (33.2\%) of the respondents had EPDS score $\geq 10$.

\section{Demographic factors}

In terms of demographic factors, no significant associations were found between EPDS score $\geq 10$ and mother's age, occupation of the mother, social support, husband support, and educational level. However, mothers suffering from marital problems before delivery were twice as likely to have PND than those without the diagnosis $(\mathrm{p}=0.018)$ and similarity those with the ongoing marital problem were also twice as likely to suffer from PND than those without the diagnosis ( $\mathrm{p}=0.038$ ) (Table 1). Lack of mother support and congenital malformations were also significantly associated with PND (Table 2).

\section{Pregnancy and birth factors}

Furthermore, no statistically significant relationships were found between postnatal depressive symptoms and: Feeding pattern of the baby, the number of previous pregnancies, history of abortion, planning of current pregnancy, and type of current delivery method. However, mothers suffering from depression during current pregnancy were

Table 1: Association between high score on the (EPDS) and socio-demographic characteristics of the newborn's mother and father

\begin{tabular}{|c|c|c|c|c|}
\hline \multirow[t]{2}{*}{ Variable } & \multirow[t]{2}{*}{ Number of participants } & \multicolumn{3}{|c|}{ Number (\%) } \\
\hline & & $E P D \geq 10$ & OR $(95 \% \mathrm{CI})$ & $\mathbf{p}$ \\
\hline \multicolumn{5}{|l|}{ Education level } \\
\hline Primary & 8 & $3(60.0)$ & $2.80(0.53-12.68)$ & 0.100 \\
\hline Secondary & 58 & 8 (13.7) & $3.65(0.61-19.2)$ & 0.071 \\
\hline Higher education & 217 & $38(21.2)$ & $0.75(0.31-1.67)$ & 0.259 \\
\hline \multicolumn{5}{|l|}{ Job } \\
\hline Not working & 106 & $18(17.0)$ & $1.03(0.53-1.99)$ & 0.453 \\
\hline Working & 164 & 27 (19.7) & & \\
\hline \multicolumn{5}{|l|}{ Income } \\
\hline$<4000$ US\$ & 10 & $1(10.0)$ & $0.51(0.02-3.43)$ & 0.298 \\
\hline$>4000$ US $\$$ & 95 & $17(17.0)$ & & \\
\hline \multicolumn{5}{|c|}{ Strong social support } \\
\hline Yes & 230 & $35(15.0)$ & $1.73(0.85-3.41)$ & 0.060 \\
\hline No & 63 & $15(23.0)$ & & \\
\hline \multicolumn{5}{|l|}{ Husband support } \\
\hline Yes & 258 & 44 (16.67) & $0.97(0.34-2.39)$ & 0.492 \\
\hline No & 36 & $6(17.05)$ & & \\
\hline \multicolumn{5}{|c|}{ Marital problems before delivery } \\
\hline Yes & 37 & $11(29.7)$ & $2.39(1.06-5.19)$ & 0.018 \\
\hline No & 260 & $39(15.0)$ & & \\
\hline \multicolumn{5}{|c|}{ Ongoing marital problem } \\
\hline Yes & 31 & $9(29.0)$ & $2.22(0.91-5.12)$ & 0.038 \\
\hline No & 258 & $40(15.5)$ & & \\
\hline
\end{tabular}


three times more likely to suffer from PND compared to those without the diagnosis $(\mathrm{p}=0.005)$. Similarly, a history of anxiety during current pregnancy made pregnant women four times more likely to suffer from PND compared to those without the diagnosis $(\mathrm{p}=0.000)$ (Table 2).

Association between high score on (EPDS) and comorbidities.

Table 3 shows that the association between PND symptoms and the mother's comorbidities including the history of diabetes, hypertension, asthma, previous psychiatric history, and family history of psychiatric illnesses.

Mothers who reported a previous history of diabetes were twice as likely to suffer from PND compared to those without the diagnosis $(\mathrm{p}=0.046)$. Similarly, mothers who had been previously diagnosed to have asthma were twice as likely to have PND compare to those without $(\mathrm{p}=0.047)$ (Table 3).

\section{DISCUSSION}

Among the most important contributors to the global burden of disease and disability are mental health problems [9]. Behavioral and mental illnesses are estimated to account for $12 \%$ of disability-adjusted lifeyears lost globally and $31 \%$ of all years lived with disability at all ages and in both sexes, according to the year 2000 estimates. Yet, more than $30 \%$ of countries have no mental health programs and over $90 \%$ have no mental health policy aimed at adolescents and children [10]. Although few studies on the prevalence of PND have been conducted

Table 2: Association between high score on the (EPDS) and perinatal history

\begin{tabular}{|c|c|c|c|c|}
\hline \multirow[t]{2}{*}{ Variable } & \multirow[t]{2}{*}{ Total number } & \multicolumn{3}{|l|}{ Number (\%) } \\
\hline & & EPD score $>10$ & OR $(95 \% \mathrm{CI})$ & $\mathbf{p}$ \\
\hline \multicolumn{5}{|c|}{ Happy with child feeding pattern } \\
\hline Yes & 244 & $42(17.2)$ & $1.25(0.46-3.36)$ & 0.346 \\
\hline No & 41 & $5(12.2)$ & & \\
\hline \multicolumn{5}{|c|}{ Depression during current pregnancy } \\
\hline Positive & 28 & $10(35.71)$ & 3.18 (1.36-7.49) & 0.005 \\
\hline Negative & 256 & $38(14.84)$ & & \\
\hline \multicolumn{5}{|c|}{ Anxiety during current pregnancy } \\
\hline Positive & 159 & $41(25.7)$ & $4.74(2.21-10.2)$ & 0.000 \\
\hline Negative & 132 & $9(6.8)$ & & \\
\hline \multicolumn{5}{|c|}{ Number of previous pregnancies } \\
\hline 0 & 12 & $1(8.3)$ & $3.75(0.58-86.26)$ & 0.102 \\
\hline 1 & 94 & $11(11.7)$ & & \\
\hline 2 & 78 & $20(25.6)$ & & \\
\hline 3 & 50 & $9(18.0)$ & & \\
\hline $4+$ & 66 & $9(13.6)$ & & \\
\hline \multicolumn{5}{|c|}{ Previous abortion } \\
\hline Yes & 77 & $9(11.6)$ & $0.58(0.48-85.16)$ & 0.087 \\
\hline No & 223 & $41(18.3)$ & & \\
\hline Total & 300 & $50(16.6)$ & & \\
\hline \multicolumn{5}{|c|}{ Planned current pregnancy } \\
\hline Yes & 177 & $28(15.8)$ & $0.87(0.46-1.66)$ & 0.337 \\
\hline No & 113 & $20(17.7)$ & & \\
\hline Total & 290 & $48(16.6)$ & & \\
\hline \multicolumn{5}{|c|}{ Type of current delivery } \\
\hline Normal & 199 & $36(18.1)$ & $1.25(0.63-2.58)$ & 0.263 \\
\hline C.S. & 87 & $13(15.0)$ & & \\
\hline Instrumental & 1 & $0(0.0)$ & & \\
\hline Total & 285 & $49(17.2)$ & & \\
\hline
\end{tabular}

EPDS: Edinburgh postnatal depression scale

Table 3: Association between high score on (EPDS) and comorbidities

\begin{tabular}{|c|c|c|c|c|}
\hline \multirow[t]{2}{*}{ Variables } & \multirow[t]{2}{*}{ Total } & \multicolumn{3}{|l|}{ Number (\%) } \\
\hline & & EPD score $>10$ & OR $(95 \% \mathrm{CI})$ & $\mathbf{p}$ \\
\hline \multicolumn{5}{|l|}{ Diabetes } \\
\hline Positive & 2 & $7(30.4)$ & $2.35(0.85-5.98)$ & 0.046 \\
\hline Negative & 275 & $43(15.6)$ & & \\
\hline \multicolumn{5}{|l|}{ HTN } \\
\hline Positive & 6 & $1(16.6)$ & $0.99(0.04-7.35)$ & 0.468 \\
\hline Negative & 292 & $49(16.7)$ & & \\
\hline \multicolumn{5}{|l|}{ Asthma } \\
\hline Positive & 6 & $1(16.6)$ & $2.49(0.84-5.82)$ & 0.047 \\
\hline Negative & 292 & $49(16.7)$ & & \\
\hline \multicolumn{5}{|c|}{ Previous psychiatric history } \\
\hline Positive & 31 & $6(19.0)$ & $2.22(0.74-6.03)$ & 0.069 \\
\hline Negative & 267 & $43(16.1)$ & & \\
\hline \multicolumn{5}{|c|}{ Family history of psychiatric disease } \\
\hline Positive & 18 & $4(22.0)$ & $2.95(0.83-10.51)$ & 0.060 \\
\hline Negative & 280 & $46(16.4)$ & & \\
\hline
\end{tabular}

EPDS: Edinburgh postnatal depression scale 
in the Arab region including Lebanon, 21\% [11]; United Arab Emirates (UAE), 22\% [12]; Tunisia, 19.2\% [13]; Jordan, 22\% [14]; and Bahrain, $37.1 \%$ [15]. However, none has been done on (SAM) living in the region including a recent study carried out in Qatar [16] which reported the prevalence of PND at $17.6 \%$. This study might have underestimated the true prevalence of PND among ethnic minorities since Qatar is a cosmopolitan and multi-ethnic society, so the true prevalence of PND might have been diluted. Furthermore, investigators in the previous study did not specifically target the (SAM) population. Therefore, firm conclusions can be drawn from these studies due to variation in research methodology used including cutoff points of applied tools, the point in time applied and cultural elements [17]. The prevalence of depressive symptoms in this study was higher than in other regional studies and sends an alarming message to policy makers and health professionals alike. Depression during pregnancy is a strong predictor of PND, and the current study showed a significant relationship between PND at 6 months and depressive symptoms reported during pregnancy. This is in contrast to the cutoff point of 1-2 weeks proposed by 0'Hara and Swain in their meta-analysis which concluded that assessment at 1 or 2 weeks postnatal was strongly associated with pregnancy depression, while assessment at 9 weeks was only weakly associated with depression [18].

Our study revealed that marital problems before and after delivery were significantly related to PND. A meta-analysis conducted by (Beck, 1996) [19] revealed significant relationships between marital dissatisfaction and prenatal anxiety.

Marital dysfunction impacts negatively on both mothers and their children, and in general, depressed females report more marital dysfunction than non-depressed females, which is demonstrated by friction, sexual dysfunction, and poor communication [20]. Furthermore, marital dysfunction persists long after the female clinically recovers from depression [21]. However, education level, income, husband support, and strong social support were not statistically significant related to PND among female participants in our study in contrast to a similar study done in a neighboring country that is sultanate of Oman [22] This may be due to the fact that the majority of our respondents were highly educated and had a high income averaging more than 4000US\$ per month, these factors make females more independent, have a higher self-esteem, and able to provide a nanny for her new offspring's which is a common practice in this part of the world. This is important because socioeconomic adversity raises the risk of negative effects not only on children but also the risk of maternal depression itself. Qatar is one of the wealthiest nations in the world. It has the second-highest gross domestic product per capita with 70,000 US dollars due to oil and natural gas [23]. The significant association between EPDS scores and depression and anxiety during current pregnancy is similar to studies done elsewhere [24]. Andersson et al. investigated the relationship between depression and anxiety during pregnancy and 6 months postpartum depression among 1.555 pregnant Swedish females. They concluded that a previous history of psychiatric illness was significantly associated with new onset of PND. Psychiatric disorders are heavily stigmatized within certain cultures, and as a result, females suffering from PND may be reluctant to seek medical help preferring to cope with their illness due to the fear of being stigmatized. Furthermore, certain cultures and religions may be misinterpreted by their own followers including Muslim females who may think that PND is their fate, and they have to accept it. Otherwise, they will be going against the will of God. As a result, females will only consult health professionals when they no longer can cope. Furthermore, the use of standardized assessment tools such EPD score may not be culturally sensitive within our culture and religion, as patients in this part of the world may deny loss of interest or lack of pleasure and even the wish of dying or feelings of self-harm as they may be regarded inappropriately as repenting against the will of God. Furthermore, having congenital malformations was a significant factor among postpartum mothers and worse still was the lack of emotional and physical support offered by the mother of postnatal females. Traditionally, the mother of the postpartum female washes the baby and at times feeds the baby which means that the mother of the newborn gets frequent respites including sleeping at night which might be a rare commodity in a mother in the postpartum period since sleep deprivation is one risk factor for postpartum depression [25]. However Sleep deprivation is rarely studied as a risk factor for PND in the medical literature. Health professionals are likely to recognize females with more severe and persistent symptoms of depression [26,27]. And although its postulated that females with preexisting diabetes are more likely to be diagnosed with PND due to a greater number of followup visits [28], this alone cannot explain the high prevalence of PND among patients with diabetes. The high prevalence of (PND) among our participants with diabetes may be due to the fact that diabetes per se increases the risk of depression by $25 \%$, as well as the fact that patients with diabetes are more likely to suffer from complications including retinopathy, neuropathy, and nephropathy. Furthermore, depression may have a bidirectional effect on chronic illnesses, such as diabetes $[29,30]$, and thus is both a risk factor for and a consequence of diabetes.

In conclusion, this study showed a high rate of depressive symptoms among (SAM) living in Qatar at 6 months postpartum, with a previous history of depression, anxiety, marital problem, diabetes, lack of mother support, history of congenital anomalies, and asthma emerging as associated risk factors. As the prevalence of PND was high, healthcare workers must be vigilant to the possibility of PND in this critical period of a mother's life. The implications of these findings are important for health policy makers. Education, screening, and intervention programs are needed and with no previous existing screening programs in Qatar, we believe that many women have suffered unnecessarily or presented late when the impact of the illness would have taken its toll. Considerable effort and resources would be needed to mount both prevention and treatment program.

\section{REFERENCES}

1. Goodman A. The development of the Qatar healthcare system: A review of the literature. Int J Clin Med 2015;6:177-85. Available from: http:// www.dx.doi.org/10.4236/ijcm.2015.63023.

2. Kaplan H, Sadock V, James B. The Summary of Psychiatry. Translated by Rezaie, F. $3^{\text {rd }}$ ed. Tehran: Arjmand Publication; 2009.

3. Almond P. Postnatal depression: A global public health perspective. Perspect Public Health 2009;129(5):221-7.

4. Gibson J, McKenzie-McHarg K, Shakespeare J, Price J, Gray R. A systematic review of studies validating the Edinburgh Postnatal Depression Scale in antepartum and postpartum women. Acta Psychiatr Scand 2009;119(5):350-64.

5. Agoub M, Moussaoui D, Battas O. Prevalence of postpartum depression in a Moroccan sample. Arch Womens Ment Health 2005;8(1):37-43.

6. Ghubash R, Abou-Saleh MT. Postpartum psychiatric illness in Arab culture: Prevalence and psychosocial correlates. Br J Psychiatry 1997;171(1):65-8.

7. Chaaya M, Campbell OM, El Kak F, Shaar D, Harb H, Kaddour A. Postpartum depression: Prevalence and determinants in Lebanon. Arch Womens Ment Health 2002;5(2):65-72.

8. Green K, Broome H, Mirabella J. Postnatal depression among mothers in the United Arab Emirates: Socio-cultural and physical factors. Psychol Health Med 2006;11(4):425-31.

9. Afifi M. Gender differences in mental health. Singapore Med J 2007;48(5):385-91.

10. World Health Organization. The World Health Report 2001. Mental Health: New Understanding, New Hope. Geneva: World Health Organization; 2001.

11. Al Hinai FI, Al Hinai SS. Prospective study on prevalence and risk factors of postpartum depression in Al-Dakhliya Governorate in Oman. Oman Med J 2014;29(3):198-202.

12. Alasoom LI, Koura MR. Predictors of postpartum depression in the Eastern Province Capital of Saudi Arabia. J Fam Med Primary Care 2014;3(2):146-50.

13. Masmoudi J, Tabelsi S, Charfeddine F, Ben Ayed B, Guermazzi M, Jaoua A. Study of the prevalence of postpartum depression among 213 Tunisian parturients. Gynecol Obstet Fertil 2008;36(7-8):782-7.

14. Mohammad KI, Gamble J, Creedy DK. Prevalence and factors associated with the development of antenatal and postnatal depression 
among Jordanian women. Midwifery 2011;27(6):e238-45.

15. Al Dallal FH, Grant IN. Postnatal depression among Bahraini women: Prevalence of symptoms and psychosocial risk factors. Eastern Med Health J 2012;18(5):439-45. Available from: http:/www.emro.who.int/ emhj-volume-18-2012/issue-5/article4.html. [Last accessed on 2013 Nov 30].

16. Burgut FT, Bener A, Ghuloum S, Sheikh J. A study of postpartum depression and maternal risk factors in Qatar. J Psychosom Obstet Gynecol 2013;34(2):90-7.

17. Rahman A, Iqbal Z, Bunn J, Lovel H, Harrington R. Impact of maternal depression on infant nutritional status and illness: A cohort study. Arch Gen Psychiatry 2004;61(9):946-52.

18. O'Hara MW, Swain AM. Rates and risk of postpartum depression: A meta-analysis. Int Rev Psychiatry 1996;8:37-54.

19. Beck C. Meta-analysis of predictors of postpartum depression. Nurs Res 1996;45(5):297-303.

20. Kung WW. The intertwined relationship between depression and marital distress: Elements of marital therapy conductive to effective treatment outcome. J Marital Fam Ther 2000;26:51-63

21. Stern G, Kruckman L. Multi-disciplinary perspectives on postpartum depression: An anthropological critique. Soc Sci Med 1983;17(15):1027-41.

22. Al Hinai FI, Al Hinai SS. Prospective study on prevalence and risk factors of postpartum depression in Al-Dakhliya governorate in Oman. Oman Med J 2014;29(3):198-202.
23. UNDP, United Nations Global Development Network. Human Development Index and Its Components. 2014. Available from: http:/ www.hdr.undp.org/sites/default/files/hdr14-report-en-1.pdf.

24. Andersson L, Sundström-Poromaa I, Wulff M, Aström M, Bixo M. Depression and anxiety during pregnancy and six months postpartum: A follow-up study. Acta Obstet Gynecol Scand 2006;85(8):937-44.

25. Dørheim SK, Bondevik GT, Eberhard-Gran M, Bjorvatn B. Sleep and depression in postpartum women: A population-based study. Sleep 2009;32(7):847-55

26. Gavin NI, Gaynes BN, Lohr KN, Meltzer-Brody S, Gartlehner G, Swinson T. Perinatal depression: A systematic review of prevalence and incidence. Obstet Gynecol 2005;106:1071-83.

27. Kelly RH, Russo J, Katon W. Somatic complaints among pregnant women cared for in obstetrics: Normal pregnancy or depressive and anxiety symptom amplification revisited? Gen Hosp Psychiatry 2001;23(3):107-13.

28. Langer N, Langer O. Comparison of pregnancy mood profiles in gestational diabetes and preexisting diabetes. Diabetes Educ 2000;26(4):667-72

29. Golden SH, Lazo M, Carnethon M, Bertoni AG, Schreiner PJ, Diez Roux AV, et al. Examining a bidirectional association between depressive symptoms and diabetes. JAMA 2008;299(23):2751-9.

30. Hirsch IB. Diabetes management. Medical clinics of North America. Clinics Review Articles. 2015 